\title{
METODOLOGIA ATIVA NO PROCESSO DE ENSINO APRENDIZAGEM DOS PROFISSIONAIS DE SAÚDE
}

\section{ACTIVE METHODOLOGY IN THE TEACHING PROCESS LEARNING OF HEALTH PROFESSIONALS}

\author{
Andreia de Bem Machado¹, Fernando Rodrigues Peixoto Quaresma²
}

RESUMO: A inovação passou a fazer parte da sociedade contemporânea com novas metodologias no fazer pedagógico do docente na área da sáude. A formação do profissional dessa área amplia-se para uma visão integral do homem. Sendo assim, o objetivo desse artigo é identificar as intersecções das metodologias ativas no processo de ensino aprendizagem na formação dos profissionais de saúde. Para tanto se realizou a análise bibliométrica, a partir de uma busca sistemática na base de dados Scopus. Como resultado, identificou-se que a pesquisa emerge no campo da medicina intersectando as discussões na área de ciências sociais, farmacologia, toxicologia e Farmacêutica.

PALAVRAS-CHAVE: Metodologia ativa. Fomação dos profissionais. Ensino-aprendizagem.

ABSTRACT: Innovation has become part of contemporary society with new methodologies in the pedagogical make of the teacher in the area of health. The training of the professional of this area extends to an integral vision of the man. Thus, the objective of this article is to identify the intersections of active methodologies in the process of teaching and learning in the training of health professionals. For that, the bibliometric analysis was performed, based on a systematic search in the Scopus database. As a result, it was identified that the research emerges in the medical field intersecting the discussions in the social sciences, pharmacology, toxicology and Pharmaceutical fields.

KEYWORDS: Active methodology. Training of professionals. Teaching and learning.

\footnotetext{
${ }_{1}^{1}$ Doutora no Programa de Pós-Graduação em Engenharia e Gestão do Conhecimento (PPEGC) na Universidade Federal de Santa Catarina (UFSC). Mestre em Educação Científica e Tecnológica (PPGECT) na Universidade Federal de Santa Catarina (UFSC). Especialista em Alfabetização na Universidade do Estado de Santa Catarina (UDESC). Graduada em Pedagogia na Faculdade de Educação (FAED) na Universidade do Estado de Santa Catarina (UDESC) Experiência na área de Educação há mais de 20 anos e com ênfase em Educação a Distância (EaD) desde de 2004, atuando principalmente nos temas: orientação de trabalho de pós-graduação a distância, planejamento, desenvolvimento, coordenação nos cursos a distância. Atualmente desenvolve trabalhos e pesquisas na área de planejamento, prática, acompanhamento e avaliação em EaD, educação corporativa, inovação, habitat de inovação, empreendedorismo, incubadora, parques tecnológicos bem como na área de Mídia e Gestão do Conhecimento. Atua também como professora formadora, palestrante, docente no ensino superior, professora orientadora e avaliadora de artigos científicos. Autora de livro e artigos científicos. andreiadebem@gmail.com

${ }^{2}$ Graduação em Enfermagem, Especialização em Saúde Pública com ênfase em Saúde Coletiva e da Família, Especialização em Docência para Educação Profissional, Mestre em Saúde Coletiva, Doutor em Ciências da Saúde Fundação de Medicina do ABC. Professor nos Cursos de Enfermagem da UFT. Tutor Residência Multiprofissional da Fundação Escola de Saúde Pública de Palmas (FESP). Experiência na área de Saúde Coletiva (ênfase em Gestão em Saúde, atuando principalmente nos seguintes temas: atenção primária, avaliação de serviços de saúde, educação em saúde e populações vulneráveis).
} 


\section{INTRODUÇÃO}

Ao longo da história nota-se que as formações dos profissionais de saúde foram pautadas em metodologias tradicionais, influenciadas por modelos fragmentados, compartimentando o conhecimento em campos altamente especializados. Isso se tornou visível nas universidades subdividido o conhecimento em departamentos, áreas, campos e os cursos em períodos modelados por disciplinas estagnadas. Sendo assim, o processo de ensino-aprendizagem, dentro desse contexto restringiu-se a repetição do conhecimento, reproduzindo-o. Dentro desse cenário o docente passa a ser um transmissor de conteúdos e o estudante um repetidor dos conteúdos, assumindo uma postura passiva e reprodutora, sem criticar ou refletir sobre esse conteúdo ou tema abordado pelo educador.

No contexto atual, em meio às tecnologias inovadoras atreladas aos meios de comunicação, o ser humano vive em um mundo ligado por redes de relações e interações dinâmicas fluidas pelas transformações constantes percebe-se a necessidade de mudanças nas instituições de ensino superior.

No cenário da saúde, surgem as discussões referentes ao processo de ensino-aprendizado visando a formação do profissional com competências e habilidades para o atendimento integral e global do ser humano, com foco na atenção que proporcionasse a cura, seja individualizada e que esteja baseada em uma causa da doença.

Para legitimar essas inquietações surge a Lei de diretrizes e bases da Educação Nacional (LDB), estimulando dentro do contexto do ensino superior o conhecimento aos problemas do mundo atual e prestação de serviço especializado à população, estabelecendo com ela uma relação de reciprocidade (BRASIL, 2009). Essas perspectivas foram implementadas pelas Diretrizes Curriculares nos cursos da área de saúde. Sendo assim, as instituições de ensino tiveram que passar por mudanças em suas práticas pedagógicas na tentativa de aproximaremse as demandas da realidade social e também motivando docentes e discentes a articular novas redes de conhecimento.
Nessa construção de novas metodologias atrelada à formação do profissional na área da saúde para a século XXI explicitadas no congresso internacional preside por Jacques Delores que são galgados em quatro pilares aprender a conhecer, aprender a fazer, aprender a viver junto e aprender a ser, que possam garantir a atenção a saúde com qualidade, eficiência e autonomia. Sendo assim, novas abordagens pedagógicas estão sendo discutidas, aperfeiçoadas e implementadas. Nesse cenário surge as metodologias ativas como alicerce no princípio teórico da freiriano (Referente a Paulo Freire, educador brasileiro, que tinha proposta pedagógica a pedagógica libertadora) da inovação. A educação na sociedade do conhecimento deve pressupor um estudante autônomo, que auto gerencie, auto governe seu processo formativo. Sendo assim, essas metodologias (CYRINO E TORALLES-PEREIRA, 2004), utilizamse da problematização como metodologia de ensino -aprendizagem, com a meta de alcançar e motivar o estudante mediante ao problema apresentado dentro do cenário educacional, relacionando sua história e passando a ressignificar as suas descobertas. Mediante a problematização, o estudante pensa e reflete sobre a informação produzindo o conhecimento tendo como objetivo solucionar os dúvidas e inquietações referente aos problemas promovendo assim o seu próprio desenvolvimento. Ao estudante observar que a nova aprendizagem é uma ferramenta significativa para ampliação de suas possibilidades e diretrizes, esse poderá galgar a liberdade e a autonomia na realização de suas escoIhas e nas tomadas de decisões.

Com base nesta contextualização, o objetivo deste estudo é identificar as intersecções das metodologias ativas no processo de ensino aprendizagem na formação dos profissionais de saúde. Para tanto, o artigo está planejado em cinco seções. A primeira acima explicitada, intitula-se introdução. Na segunda seção, apresentam-se os procedimentos da pesquisa. Na terceira seção, mostra-se de modo detalhado o resultado bibliométrico de pesquisa baseada no cenário das publicações científicas desta área. Na quarta seção, são explicitadas as considerações finais. Por último, na quinta seção, elencam-se as referências utilizadas para construir este artigo. 


\section{Caminho Metodológico}

Para atender a problemática da pesquisa proposta neste estudo, empregou-se uma metodologia classificada como exploratório-descritiva. Esta metodologia tem o objetivo de delinear o tema e aumentar a familiaridade dos pesquisadores com o fato, além de esclarecer os conceitos inerentes ao tema em estudo (MARCONI; LAKATOS, 2010).

Como método de pesquisa da literatura, utilizouse a busca sistemática em uma base de dados on-line, seguida de uma análise bibliométrica dos resultados. A bibliometria é uma metodologia oriunda das ciências da informação que utiliza métodos matemáticos e estatísticos para mapear documentos e padrões de publicação a partir de registros bibliográficos armazenados em bases de dados (FEATHER; STURGES, 2003; SANTOS; KOBASCHI, 2009). Desse modo, a metodologia bibliométrica possibilita, para os autores citados, contagens relevantes como: produção por região; temporalidade das publicações; pesquisas por área do conhecimento; contagem de literatura relacionada à citação do estudo; fator de impacto de uma publicação científica. Estas contagens permitem visualizar dados matemáticos e estatísticos que corroboram para a sistematização do resultado de uma pesquisa e a minimização da ocorrência de intersecções ao se olhar para um determinada temática.

\section{Procedimentos para coleta de dados}

Para a análise bibliométrica, o estudo foi elaborado em três etapas distintas: planejamento, coleta e resultado. Estas etapas aconteceram de modo integrado e síncrono para responder à pergunta norteadora da pesquisa: Quais as intersecções entre as metodologias ativas e formação de profissionais na área de saúde?

O planejamento iniciou-se no mês de setembro de 2016, quando a pesquisa foi efetivada. No escopo do planejamento, foi estipulada como relevante para o domínio da pesquisa a base de dados Scopus (<http://www.scopus.com>), devido a sua relevância no meio acadêmico, seu caráter interdisciplinar, sua atualidade e também por ser uma das maiores bases de resumos e referências bibliográficas de literatura científica revisada por pares.

Considerando-se o problema de pesquisa, delimitou-se, na fase de planejamento, os termos de busca, a saber: "active teaching-learning methodologies" and training of "health professionals". Como princípio básico para a busca, optou-se pela utilização dos termos nos campos "title", "abstract" e "keyword", sem restrição temporal, de idioma ou outra qualquer que possa limitar o resultado.

\section{Procedimentos para análise de dados}

Com base no planejamento da pesquisa, a coleta de dados recuperou um total de 5 trabalhos indexados, com o primeiro registro datado de 2010 e o último de 2014.

Como resultado desta coleta de dados, identificou-se que os trabalhos foram escritos por 25 autores, vinculados a 13 instituições provenientes do Brasil. Foram utilizadas 31 palavras-chave para identificar e indexar as publicações, que se apresentam distribuídas em 4 áreas do conhecimento e 1 tipo de publicação. O quadro 1 apresenta o resultado da coleta de dados numa análise bibliométrica geral dos resultados obtidos na base de dados Scopus.

\begin{tabular}{|l|l|}
\hline Base de dados & $\begin{array}{l}\text { Scopus } \\
\text { "active teaching-learning } \\
\text { methodologies" and } \\
\text { training of "health } \\
\text { professionals" } \\
\text { "title", "abstract", "keyword" }\end{array}$ \\
\hline Campos de busca & 5 \\
\hline Total de trabalhos & 5 \\
\hline recuperados & 25 \\
\hline Autores & 13 \\
\hline Instituições & 1 \\
\hline Países & 31 \\
\hline Palavras-chave & 4 \\
\hline Áreas do conhecimento & 4 \\
\hline Tipos de publicação & 1 \\
\hline
\end{tabular}

Quadro 1 - Dados bibliométricos gerais obtidos na base de dados Scopus

Fonte: Elaborado pelos autores (2016). 
O universo de 5 trabalhos científicos compõe a amostra para uma análise bibliométrica geral das publicações na área de ciências humanas, farmacologia, toxicologia e Farmacêutica, sem limitações específicas, o que permite tecer o estado da arte do tema a partir da base de dados consultada.

Para a apreciação dos resultados de maneira mais aprofundada na análise bibliométrica, fez-se a exportação do resultado para um software de gerenciamento bibliográfico denominado EndNoteWeb (software baseado na Web que contribui com o trabalho do pesquisador durante o processo de escrita de sua pesquisa.). Assim, as informações relevantes foram classificadas de acordo com: distribuição temporal; principais autores, instituições e países; tipo de publicação na área; principais palavras-chave e trabalhos mais referenciados.

\section{Resultados e discussão}

Num primeiro momento, analisou-se a distribuição temporal dos trabalhos, o que permitiu identificar que a primeira publicação sobre o tema tem registro de 2010 com somente um trabalho neste ano e nos anos de 2012 e 2013. Nos anos de 2011 e 2015 não houve publicações e no ano de 2014 houve 2 publicações. Para melhor visualização da frequência de publicações, elaborou-se o gráfico 1.

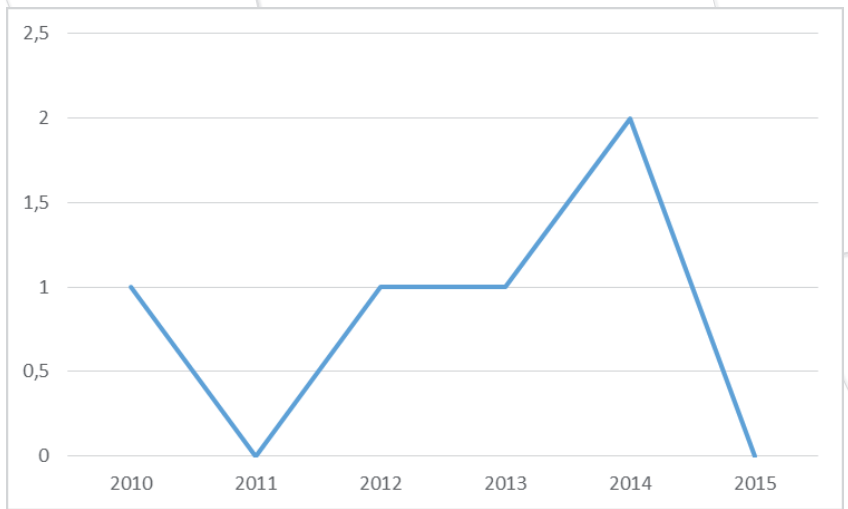

Gráfico 1 - Distribuição dos trabalhos por ano Fonte: Elaborado pelos autores (2016).

Foi identificado um artigo como pioneiro, publicado em 2010 intitulado Multiprofessional health-related graduate courses: Results from experiences using active methodologies dos autores Maria José Sanches
Marin; Romeu Gomes; Marilda Marques Luciano Marvulo; Elisabete Medeiros Primo; Pedro Marco Karan BarbosaV e Suelaine Druzian. Nesse artigo os autores avaliam os resultados de uma experiência em um curso de pós-graduação ancoradas em metodologias ativas, a partir da visão de seus egressos. 0 artigo explicita que essas contribuem para o processo de ensino aprendizagem formando profissionais, em nível de pós-graduação, que estão alinhados à atual política nacional de saúde, visando ao atendimento com qualidade, autonomia e eficiência. Segundo os autores, essa metodologia envolve um fazer pedagógico voltado a práticas inovadoras onde

A prática interdisciplinar é revestida de grande complexidade, por exigir dos profissionais nova forma de agir no seu cotidiano, além de implicar mudanças nas relações de poder entre os profissionais de saúde para que se amplie efetivamente a autonomia dos usuários e coletividades. (MARIN; MARVULO; BARBOSA; DRUZIAN, 2010, p.5)

No ano de 2012 foi publicado o artigo intitulado The construction of collective portfolios in traditional curriculums: An innovative approach in teaching-learning de autoria de Rosângela Minardi Mitre Cotta; Luciana Saraiva da Silval; Lílian Lelis Lopes ; Karine de Oliveira Gomes; Fernanda Mitre Cotta; Regina Lugarinho e Sandra Minardi Mitre. Os autores abordam a utilização do portfólio, bem como da metodologia ativa na disciplina Políticas de saúde. Percebe-se que a metodologia ativa é a capacidade de despertar nos alunos a aptidão para inter-relacionar o conhecimento anterior ao novo. Assim Cotta et al 2012, explicita que a aprendizagem é um processo ativo de construção do conhecimento, quando há um conteúdo significante, ou seja, quando o estudante interage com elementos novos e os já existentes na sua estrutura cognitiva.

Com a utilização da metodologia ativa e também dos portfolios na disciplina de Políticas de Saúde da Universidade Federal de Viçosa (UFV), os autores observaram que se gerou valorização do trabalho em equipe, a busca pela reflexão e discussões dos problemas visando soluções através de práticas de gestão do conhecimento. 
No ano de 2013, foi publicado o artigo intitulado Current debates on humanization and health: Who are we? de autoria Rosângela Minardi Mitre Cotta; Roberta Sena Reis; Aline Aparecida de Oliveira Campos; Andréia Patrícia Gomes; Vanderson Espiridião Antonio; Rodrigo Siqueira-Batista. Nesse artigo os autores discutem sobre a formação dos profissionais da área de saúde bem como as metodologias de ensino aprendizagem que são utilizadas no contexto do processo formativo desses profissionais, dentre elas destaca-se a metodologia ativa. Para Cotta et al 2013, a metodologia ativa atrelada a problematização do conhecimento são estratégias inovadoras que estimulam discussões e analise crítica dos temas propostos pelos professores e são fundamentais para o aprendizado ativo gerando autonomia no estudante.

Já no ano de 2014 foram publicados 2 artigos, um intitulado Pharmacist education in Brazil from a clinical perspective dos autores Rodrigo Batista de Almeida, Dayanna Hartmann Cambruzzi Mendes, Pablo Alfredo Dalpizzol. Esse artigo tem o objetivo de discutir a interação entre o ensino e a prática farmacêutica voltada para a formação clínica. Dentro desse tema foram também discutidas práticas pedagógicas interativas e inovadoras que proporcionam resultados qualitativos na farmacoterapia, melhorando a qualidade de vida dos usuários de medicamentos. $\mathrm{E}$ também foi publicado o artigo intitulado Active teaching-learning methodologies and popular education: Agreements and disagreements in the context of health professionals' education de autoria Eduardo Simon, Edineide Jezine, Eymard Mourão Vasconcelos e Katia Suely Queiroz Silva Ribeiro. Esse artigo explicita as metodologias dentro do processo de ensino aprendizagem como alicerces para a formação com qualidade, autonomia e eficiência dos profissionais de saúde. Nessa abordagem são elencados a metodologia ativa, como uma ferramenta pedagógica na formação do profissional em saúde para a sociedade contemporânea baseada no conhecimento.

Com base no gráfico 1, que ilustra a frequência de publicações sobre o tema em estudo neste artigo, pode-se perceber que a pesquisa na área é relevante e que, de modo geral, ainda são incipientes as discussões relacionadas a metodologia ativa e formação dos profissionais em saúde. Destacando o Brasil como o único país com publicações referentes a essa temática.
Dos 5 trabalhos filtrados na busca, observa-se uma variada lista de autores, instituições no Brasil que se destacam no que tange ao tema "metodologia ativa e formação dos profissionais em saúde". Observou-se que 25 autores podem ser considerados como referência em publicações sobre o tema. Percebe-se que há grupos discutindo sobre essa área, pois há 5 publicações no Brasil e essas foram realizadas em parcerias, sendo cada uma delas publicadas com o nome de 5 autores. Sendo que esses são oriundos de grupos de pesquisa sobre metodologia ativa e formação de profissionais em saúde. Buscando-se organizar estes dados, elaborou-se o quadro 2 , com autores de destaques na área, quantidade de artigos publicados, instituição à qual estão afiliadas ao Brasil e Espanha.

\begin{tabular}{|c|c|c|c|}
\hline Autor & $\begin{array}{c}\text { Quantidade } \\
\text { de } \\
\text { publicações }\end{array}$ & Afiliação & País \\
\hline $\begin{array}{l}\text { Cotta, } \\
\text { Rosângela } \\
\text { Minardi Mitre } \\
\text { Itre }\end{array}$ & 2 & $\begin{array}{l}\text { Universidade } \\
\text { Federal de Viçosa, } \\
\text { Departamento de } \\
\text { Nutrição e Saúde }\end{array}$ & Brasil \\
\hline $\begin{array}{l}\text { Antonio, } \\
\text { Vanderson } \\
\text { Espiridião }\end{array}$ & 1 & $\begin{array}{l}\text { Universidade } \\
\text { Federal de Viçosa, } \\
\text { Departamento de } \\
\text { Nutrição e Saúde }\end{array}$ & Brasil \\
\hline $\begin{array}{l}\text { Barbosa, P. } \\
\text { M K }\end{array}$ & 1 & $\begin{array}{l}\text { Hospital de Clínicas } \\
\text { de Marília, Serviço } \\
\text { de Enfermagem, }\end{array}$ & Brasil \\
\hline $\begin{array}{l}\text { Campos, } \\
\text { Aline } \\
\text { Aparecida de } \\
\text { Oliveira }\end{array}$ & 1 & $\begin{array}{l}\text { Universidade } \\
\text { Federal de Viçosa, } \\
\text { Departamento de } \\
\text { Nutrição e Saúde, } \\
\text { Viçosa }\end{array}$ & Brasil \\
\hline $\begin{array}{l}\text { Cotta, } \\
\text { Fernanda } \\
\text { Mitre }\end{array}$ & 1 & $\begin{array}{l}\text { Faculdade de } \\
\text { Medicina de } \\
\text { Barbacena }\end{array}$ & Brasil \\
\hline $\begin{array}{l}\text { Dalpizzol, } \\
\text { Pablo Alfredo }\end{array}$ & 1 & $\begin{array}{l}\text { Instituto Federal } \\
\text { do Paraná (IFPR), } \\
\text { Curitiba }\end{array}$ & Brasil \\
\hline $\begin{array}{l}\text { De Almeida, } \\
\text { Rodrigo } \\
\text { Batista }\end{array}$ & 1 & $\begin{array}{l}\text { Instituto Federal } \\
\text { do Paraná (IFPR), } \\
\text { Curitiba }\end{array}$ & Brasil \\
\hline $\begin{array}{l}\text { Druzian, } \\
\text { Suelaine }\end{array}$ & 1 & $\begin{array}{l}\text { FAPESP Technical } \\
\text { Grantholder, Marilia }\end{array}$ & Brasil \\
\hline $\begin{array}{l}\text { Gomes, } \\
\text { Andréia } \\
\text { Patrícia } \\
\text { Atricia }\end{array}$ & 1 & $\begin{array}{l}\text { Universidade } \\
\text { Federal de Viçosa, } \\
\text { Departamento } \\
\text { de Medicina e } \\
\text { Enfermagem, } \\
\text { Viçosa }\end{array}$ & Brasil \\
\hline
\end{tabular}




\begin{tabular}{|c|c|c|c|}
\hline $\begin{array}{l}\text { Gomes, } \\
\text { Romeu L E }\end{array}$ & 1 & $\begin{array}{l}\text { Fundação Oswaldo } \\
\text { Cruz, Instituto } \\
\text { Nacional de } \\
\text { Saúde da Mulher } \\
\text { da Criança e } \\
\text { do Adolescente } \\
\text { Fernandes Figueira, } \\
\text { Rio de Janeiro }\end{array}$ & Brasil \\
\hline $\begin{array}{l}\text { Jezine, } \\
\text { Edineide }\end{array}$ & 1 & $\begin{array}{l}\text { Universidade } \\
\text { Federal da Paraiba, } \\
\text { Departamento } \\
\text { de Promoção } \\
\text { da Saúde, Joao } \\
\text { Pessoa }\end{array}$ & Brasil \\
\hline $\begin{array}{l}\text { Lopes, Lílian } \\
\text { Lelis }\end{array}$ & 1 & $\begin{array}{l}\text { Universidade } \\
\text { Federal de Viçosa, } \\
\text { Departamento de } \\
\text { Nutrição e Saúde, } \\
\text { Viçosa }\end{array}$ & Brasil \\
\hline $\begin{array}{l}\text { Lugarinho, } \\
\text { Regina }\end{array}$ & 1 & $\begin{array}{l}\text { Universidade } \\
\text { Federal do Estado } \\
\text { do Rio de Janeiro, } \\
\text { Rio de Janeiro }\end{array}$ & Brasil \\
\hline $\begin{array}{l}\text { Marin, } \\
\text { Maria José } \\
\text { Sanches }\end{array}$ & 1 & $\begin{array}{l}\text { Escola de Medicina } \\
\text { de Marilia, São } \\
\text { Paulo }\end{array}$ & Brasil \\
\hline $\begin{array}{l}\text { Marvulo, M. } \\
\text { M L }\end{array}$ & 1 & $\begin{array}{l}\text { Faculdade de } \\
\text { Medicina de Marilia, } \\
\text { Marilia }\end{array}$ & Brasil \\
\hline $\begin{array}{l}\text { Mendes, } \\
\text { Dayanna } \\
\text { Hartmann } \\
\text { Cambruzzi }\end{array}$ & 1 & $\begin{array}{l}\text { Instituto Federal } \\
\text { do Paraná (IFPR), } \\
\text { Curitiba }\end{array}$ & Brasil \\
\hline $\begin{array}{l}\text { Mitre, Sandra } \\
\text { Minardi }\end{array}$ & 1 & $\begin{array}{l}\text { Universidade } \\
\text { Federal de Minas } \\
\text { Gerais, Belo } \\
\text { Horizonte }\end{array}$ & Brasil \\
\hline $\begin{array}{l}\text { Primo, } \\
\text { Elisabete } \\
\text { Medeiros }\end{array}$ & 1 & $\begin{array}{l}\text { Serviço de } \\
\text { Desenvolvimento de } \\
\text { Pessoal e Ouvidoria }\end{array}$ & Brasil \\
\hline $\begin{array}{l}\text { Reis, } \\
\text { Roberta } \\
\text { Sena }\end{array}$ & 1 & $\begin{array}{l}\text { Universidade } \\
\text { Federal de Viçosa, } \\
\text { Departamento de } \\
\text { Nutrição e Saúde, } \\
\text { Viçosa }\end{array}$ & Brasil \\
\hline $\begin{array}{l}\text { Ribeiro, } \\
\text { Kátia Suely } \\
\text { Queiroz Silva }\end{array}$ & 1 & $\begin{array}{l}\text { Universidade } \\
\text { Federal da Paraiba, } \\
\text { Departamento de } \\
\text { Fisioterapia, Joao } \\
\text { Pessoa }\end{array}$ & Brasil \\
\hline $\begin{array}{l}\text { Simón, } \\
\text { Eduardo }\end{array}$ & 1 & $\begin{array}{l}\text { Hospital } \\
\text { Universitario La Fe, } \\
\text { Valencia, Spain }\end{array}$ & Espanha \\
\hline
\end{tabular}

\begin{tabular}{|l|c|l|c|}
\hline $\begin{array}{l}\text { Sîqueira- } \\
\text { Batista, } \\
\text { Rodrigo }\end{array}$ & 1 & $\begin{array}{l}\text { Curso de Medicina, } \\
\text { Faculdade Dinâmica } \\
\text { do Vale do Piranga, } \\
\text { Belo Horizonte }\end{array}$ & Brasil \\
\hline $\begin{array}{l}\text { Vasconcelos, } \\
\text { Eymard } \\
\text { Mourão }\end{array}$ & 1 & $\begin{array}{l}\text { Universidade } \\
\text { Federal da Paraiba, } \\
\text { Departamento } \\
\text { de Promoção } \\
\text { da Saúde, Joao } \\
\text { Pessoa }\end{array}$ & Brasil \\
\hline $\begin{array}{l}\text { Da Silva, } \\
\text { Luciana } \\
\text { Saraiva M }\end{array}$ & 1 & $\begin{array}{l}\text { Universidade } \\
\text { Federal de Vicosa, } \\
\text { Departamento de } \\
\text { Nutrição e Saúde, } \\
\text { Viçosa }\end{array}$ & Brasil \\
\hline $\begin{array}{l}\text { De Oliveira } \\
\text { Gomes, } \\
\text { Karine }\end{array}$ & 1 & $\begin{array}{l}\text { Universidade } \\
\text { Federal da } \\
\text { Bahia, Instituto } \\
\text { Multidisciplinar em } \\
\text { Saúde, Salvador }\end{array}$ & \\
\hline
\end{tabular}

Quadro 2 - Autores com maior número de publicações na área, com suas afiliações e seus países Fonte: Elaborado pelos autores (2016).

Com base no quadro 2, observa-se que a instituição com mais profissionais discutindo e publicando sobre essa temática é na Universidade Federal de Viçosa, Departamento de Nutrição e Saúde em Viçosa Minas Gerais, com sete profissionais filiados nessa instituição.

Com base no levantamento geral, foi possível analisar-se ainda o tipo de documento das pesquisas na área de metodologia ativa e formação dos profissionais em saúde, que se concentrou apenas em artigos.

A partir da análise bibliométrica, com base no grupo de trabalhos recuperados na base de dados Scopus, foi possível identificar um total de 31 palavraschave diferentes. O destaque ficou com a palavra-chave artigo com 3 ocorrências, seguidas da palavra metodologias de ensino-aprendizagem ativas com duas ocorrências, Brasil, educação em saúde, humana, metodologia ativa de educação, aprender com duas ocorrências cada uma e farmácia clínica, currículo com apenas uma citação. Essas são as palavras destaques entre outros que apareceram como palavras nesse cenário de publicação.

$\mathrm{Na}$ análise das palavras-chave, percebe-se que a discussão sobre metodologia ativa e formação dos profissionais em saúde converge com os temas "metodologias de ensino aprendizagem", "educação em 
saúde", "educação" e "humana". O debate envolve ainda a preocupação com as políticas públicas voltadas a educação do profissional na área de saúde, trazendo à tona áreas como ciências sociais e metodologias de ensino aprendizagem, e abarca questões como aprender a aprender, aprender a ser, aprender a conviver e aprender a fazer.

\section{Considerações finais}

Tratar das metodologias ativas e formação dos profissionais em saúde implica debater aspectos relacionados ao processo didático pedagógico de ensinar e aprender, atrelados a ferramentas inovadoras que formem um sujeito crítico e autônomo para a efetivo trabalho no cenário da saúde, garantindo qualidade no atendimento e na prestação de serviços nesse setor.

O mapeamento científico da produção relacionada ao tema "metodologias ativas e formação dos profissionais em saúde", feito na base de dados Scopus, permitiu uma análise bibliométrica do tema que descrevesse as principais discussões da contemporaneidade e a interseção com áreas sem recorte temporal. Como resultado, identificou-se que a pesquisa emerge no campo multidisciplinar, intersectando as discussões sobre medicina, farmacologia, taxologia, enfermagem e saúde. Finalmente, detectou-se que as discussões sobre pessoas com metodologia ativas atreladas formação dos profissionais em saúde carecem de estudos, principalmente no Brasil.

\section{REFERÊNCIAS}

BRASIL. Lei de diretrizes e bases da educação nacional 9. 394, 20 de dezembro de 1996. 2009. Disponível em: <http://www.planalto.gov.br/ccivil_03/leis/ L9394.htm>. Acesso em: 07 set. 2016.

COTTA, R. Minardi.M.; Silva, L S ; Lopes, L L ; GOMES, Karine O ; Cotta, Fernanda Mitre ; LUGARINHO, Regina ; Mitre, Sandra M. The construction of collective portfolios in traditional curriculums: An innovative approach in teaching-learning. Ciênc. saúde coletiva, v. 17, p. 787-796, 2012. Disponível em: http://www.scielo.br/scielo.php?script=sci arttext\&pi$\mathrm{d}=\mathrm{S} 1413-81232012000300026$, Acesso em: $22 \mathrm{fev}$. 2019.

COTTA, R. Minardi.M.; REIS, Roberta Sena; CAMPOS, AAO; GOMES, Andréia Patrícia; Antônio, Vanderson E; BATISTA, Rodrigo Siqueira. Current debates on humanization and health: who are we? Ciênc. saúde coletiva, v. 18, p. 171-9, 2013. Disponível em: http://www.scielo.br/scielo.php?script=sci_arttext\&pi$\mathrm{d}=\mathrm{S} 1413-81232013000100018$. Acesso em: $22 \mathrm{fev}$. 2019.

CYRINO EG, TORALLES-PEREIRA ML. Trabalhando com estratégias de ensino-aprendizado por descoberta na área da saúde: a problematização e a aprendizagem baseada em problemas. Cad. Saúde Pública, 2004;20(3):780-788.

LAKATOS, E. M.; MARCONI, M. de A. Fundamentos de metodologia científica. São Paulo: Atlas, 2010. MARIN, M. J. S.; Gomes, R; MARVULO, M. M. L.; Primo, E. M.; BARBOSA, Pedro Marco K; DRUZIAN, S. P. Multiprofessional health-related graduate courses: results from experiences using active methodologies. Interface (Botucatu. Impresso), v. 14, p. 303-314, 2010. SANTOS, R. N. M.; KOBASHI, N. Y. Bibliometria, cientometria, infometria: conceitos e aplicações. Tendências da Pesquisa Brasileira em Ciência da Informação, Brasília, v. 2, n. 1, p. 155-172, 2009. Disponível em: <http://inseer.ibict.br/ancib/index.php/tpbci/ article/viewArticle/21>. Acesso em: 16 out. 2014. 